\title{
Trato del docente y el rendimiento académico en las prácticas clínicas de los estudiantes de enfermería de una Universidad Privada de Lima, 2015
}

\author{
Treatment of the teacher and academic performance in clinical practice of the nursing students in a \\ private University in Lima, 2015
}

\author{
Katherine Mescua Fasanando ${ }^{1 *}$, Deysy Calderón Tafur², Ángela Paredes Aguirre ${ }^{3}$
}

\begin{abstract}
RESUMEN
Objetivo: Determinar la relación entre el trato del docente y el rendimiento académico en las prácticas clínicas de los estudiantes de enfermería de una universidad privada de Lima, 2015. Metodología: El enfoque cuantitativo, de diseño no experimental, de corte transversal y de tipo correlacional. El muestreo fue no probabilístico de tipo intencional y la muestra estuvo conformada por 130 estudiantes de enfermería. Se utilizó el cuestionario modificado sobre "Trato del docente de enfermería" de Muñasqui y para el rendimiento académico se utilizó el registro de notas de los estudiantes. Resultados: Los resultados indicaron que existe relación inversa baja entre el trato del docente y el rendimiento académico en las prácticas clínicas $(\mathrm{t}=-.189 ; \mathrm{p}<, 05)$, al igual que en las dimensiones de empatía $(\mathrm{t}=-.145 ; \mathrm{p}<, 05)$ y respeto $(\mathrm{t}=-.137 ; \mathrm{p}<, 05)$, menos en la dimensión comunicación ( $\mathrm{t}=$ $.129 ; \mathrm{p}>, 05)$. Respecto al trato del docente se encontró que el 46,9\% es regular con inclinación a lo malo. Por otro lado, el 51,5\% presentó un rendimiento académico bueno, seguido de un 37,7\% regular y un 10,8\% excelente. Conclusión: Existe relación significativa entre el trato del docente y el rendimiento académico en las prácticas clínicas de los estudiantes de enfermería.
\end{abstract}

Palabras clave: Prácticas clínicas, trato, práctica del docente de enfermería y rendimiento académico.

\begin{abstract}
Objective: Determine the relationship between the treatment of the teacher and academic performance in clinical practice of the nursing students in a private university in Lima, 2015. Methodology: Quantity approach, nonexperimental design, transversal cut and correlational type. Sampling was non probabilistic type intentional and the sample was composed by 130 students of nursing. A questionnaire modified about "Treatment of nursing teacher" was used by Muñasqui. The registration of student's grades was used to the academic performance. Results: The results indicate that there is a low inverse relationship between the treatment of the teacher and the academic performance in clinical practices, $(\mathrm{t}=-.189 ; \mathrm{p}<, 05)$ and is similar in dimensions of empathy $(\mathrm{t}=-.145$; $\mathrm{p}<, 05)$ and respect $(\mathrm{t}=-.137 ; \mathrm{p}<, 05)$, less in the communication dimension $(\mathrm{t}=-.129 ; \mathrm{p}>, 05)$. According to the treatment of teachers it was found $46.9 \%$ concerns a regular deal with inclination to wrong. On the other hand, $51.5 \%$ presents a good academic performance, followed by a regular $37.7 \%$ and $10.8 \%$ excellent. Conclusion: There is a significant relationship between the treatment of the teacher and academic performance in clinical practice for students of nursing.
\end{abstract}

Keywords: Clinical practice, treatment, nursing, nursing teachers practice, academic performance.

${ }^{1}$ Coordinadora de Especialidades en Enfermería, UPG, Universidad Peruana Unión, Lima, Perú.

${ }^{2}$ Licenciada en Enfermería, Clínica Good Hope, Miraflores, Perú.

${ }^{3}$ Doctora en Educación, Facultad de Ciencias de la Salud, Universidad Peruana Unión, Lima, Perú. 


\section{INTRODUCCIÓN}

Para que los estudiantes alcancen el logro académico se necesita un buen docente, el cual debe reunir características que faciliten el proceso del aprendizaje, por lo tanto, el docente no solamente debe poseer conocimientos científicos sino también habilidades sociales que incentiven el buen clima en la formación académica.

La docencia en enfermería es un área del conocimiento educativo que tiene un gran valor en la práctica profesional, formando parte de una de las funciones que involucra esta profesión, debido a que el enfermero tendrá que asumir siempre el rol docente en el área donde se desempeñe (Yarasca, 2012).

Cuando el enfermero se centra en la práctica asistencial identificamos como benefactor del cuidado al paciente, pero cuando cumple la función docente el benefactor principal pasan a ser inmediatamente los estudiantes que están a su cargo, entonces el trato que llega a brindar a ambos protagonistas debe ser el mismo, basado en los principios axiológicos como fruto de un trato humanizado. Hay que dejar en claro que la educación es un proceso continuo que acompaña y atraviesa toda nuestra vida, por lo tanto es innato en el hombre. (Cortez, 2008).

Para Muñasqui (2007), el enfermero que asume el rol docente, debe mostrar un dominio elevado del curso a enseñar, preocuparse por el buen trato con sus alumnos, prepararse en pedagogía si fuera necesario, definir los objetivos y acciones que espera que sus estudiantes realicen, profundizar sus conocimientos, propiciar participación del equipo de salud y mantener una adecuada comunicación con los estudiantes. Por lo tanto, la actuación del docente de prácticas se transforma en un elemento importante, al integrarse con los estudiantes en los campos clínicos proporciona a través de sus conocimientos y actitudes, las bases y el apoyo necesario para el desarrollo de habilidades que conducen a la formación de un profesional competente, a fin de poder actuar en beneficio de la sociedad como futuros profesionales de enfermería (Bettancourt, Muñoz, Aparecida y Merighi, 2011).

No obstante, no debemos olvidar que en diversas universidades los docentes de aula no son los mismos del campo clínico. En el área clínica el estudiante aplica y transforma los conocimientos teóricos aprendidos durante las clases, a través de acciones prácticas de cuidado en el área hospitalaria, las cuales son apoyadas por el docente de prácticas (Scherer y Scherer, 2007).
En un estudio realizado en España, se identificaron que los elementos primordiales que favorecen el aprendizaje de los estudiantes de enfermería en el campo clínico son las habilidades docentes para la enseñanza, y las relaciones interpersonales entre los docentes y los estudiantes. A veces las actitudes del docente de prácticas clínicas no son aceptados por los estudiantes porque aseguran que no reciben un buen trato por ellos, ya que el educador se basa en lo teórico-práctico, pero deja muchas veces a un lado la relación interpersonal-psico-emocional con el estudiante (Scherer y Scherer, 2007).

En una investigación realizada en Perú, acerca de la percepción que tiene el estudiante de enfermería sobre el trato del docente de prácticas clínicas, se observó que la mayoría de ellos consideran que el respeto es de regular a deficiente porque los docentes juzgan sus actitudes en presencia de sus compañeros. Por otro lado, hacen gestos desagradables cuando se expresan, son incomprensivos y muestran desinterés por el estado emocional (Muñasqui, 2007).

La mala relación con el docente influye en el rendimiento académico de los estudiantes. El cual, es considerado como el resultado de distintos factores multicausales que inciden en la respuesta académica, donde interactúan elementos sociodemográficos, psicosociales, pedagógicos, institucionales y socioeconómicos; entre ellos, elementos tan variados como: la motivación, la ansiedad, la autoestima, la percepción del clima académico, el entusiasmo, el docente, el sentido de propósito, entre otros (Lescano, 2002, Montero y Villalobos, 2004).

Las actitudes que llegan a tomar los enfermeros en su rol docente frente a sus estudiantes pueden repercutir de manera positiva o negativamente en su desenvolvimiento evidenciándose directamente en el rendimiento académico llegándose a generar una incertidumbre entre la relación significativa que ambos podrían tener.

Es por eso, que en este estudio se pretende encontrar la relación entre el trato del docente y el rendimiento académico en las prácticas clínicas de los estudiantes de enfermería de una Universidad Privada de Lima. Siendo necesario para generar nuevas estrategias metodológicas para la adquisición de conocimientos científicos y prácticos, y estimular fundamentalmente el desarrollo de aptitudes y valores. 
Trato del docente y el rendimiento académico en las prácticas clínicas de los estudiantes de enfermería de una Universidad Privada de Lima, 2015

\section{MATERIAL Y MÉTODOS}

Esta investigación es de diseño no experimental, puesto que no se manipulan las variables de investigación, de enfoque cuantitativo porque utiliza el análisis estadístico para contestar la pregunta de investigación y probar la hipótesis establecida. Además es de corte transversal porque se recogió los datos en un solo momento. De tipo correlacional ya que mide la relación entre trato del docente y rendimiento académico.

La población estuvo constituida por 284 estudiantes de enfermería. Se utilizó un muestreo no probabilístico de tipo intencional, cumpliendo con los criterios de inclusión y exclusión, donde se obtuvo como muestra a 130 estudiantes de enfermería. El instrumento utilizado fue: "trato de docente de prácticas clínicas" elaborado por Muñasqui en el 2007 el cual fue modificado por las investigadoras y consta de 33 ítems, que se desdoblan en tres dimensiones: Comunicación, empatía y respeto. La fiabilidad del instrumento se obtuvo mediante la validación por juicio de expertos y la prueba de confiabilidad Alfa de Crombach, cuyo resultado indico una excelente confiabilidad ( $\alpha=0.877)$.

El método de recolección de datos utilizado fue la encuesta, el cual fue aplicado de forma individual, con una duración de 15 minutos aproximadamente, previo consentimiento informado.

Para evaluar el rendimiento académico, se solicitaron las notas de las prácticas clínicas de los estudiantes de enfermería y se creó una escala vigesimal para medir el rendimiento académico según los ponderados, el cual fue validado por juicio de expertos. El software utilizado para el análisis de la información fue el estadístico SPSS versión 23 para Windows ${ }^{\circledR}$, el cual permitió codificar la encuesta sobre el trato del docente y las notas de los estudiantes de enfermería. Posteriormente, se realizó un análisis descriptivo de ambas variables según su escala de medición y como paso final, se analizó la correlación entre ambas variables ordinales a través del coeficiente de correlación Tau-b de Kendall.

\section{RESULTADOS}

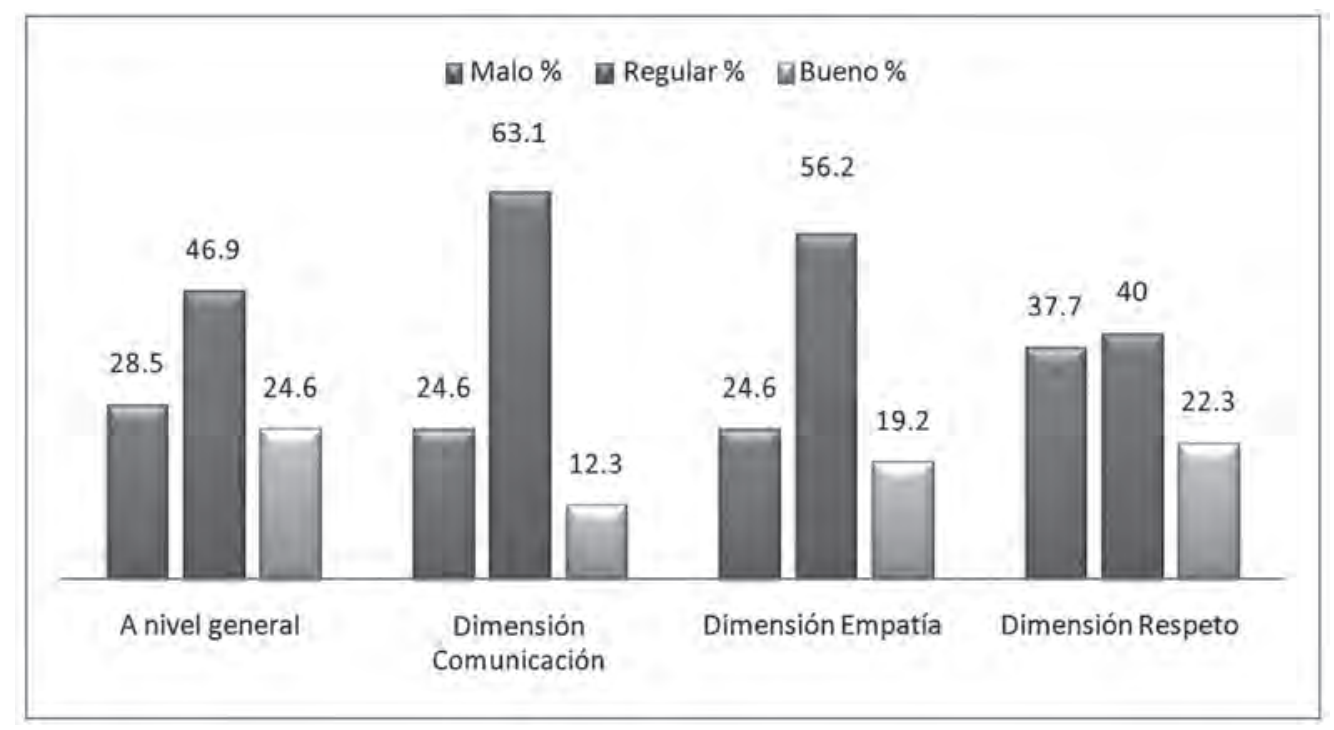

Figura 1

Tipo de trato del docente de prácticas clínicas de una universidad privada de Lima, 2015.

En la figura 1 se observa que a nivel general el trato del docente de prácticas clínicas es regular con el 46,9\%, mientras que el $28,5 \%$ de estudiantes dice que es malo y el $24,6 \%$ es bueno. Así mismo el 63,1\% de estudiantes refieren una comunicación regular por parte del docente de prácticas clínicas, el 24,6\% malo y el $12,3 \%$ bueno. De la misma manera en la dimensión empatía, el 56,2\% refieren una empatía regular del docente de prácticas clínicas, el 24,6\% malo y el 19,2\% bueno. Por último el $40 \%$ dicen que el docente tiene un respeto regular, el $37,7 \%$ malo y el 22,3\% bueno. 


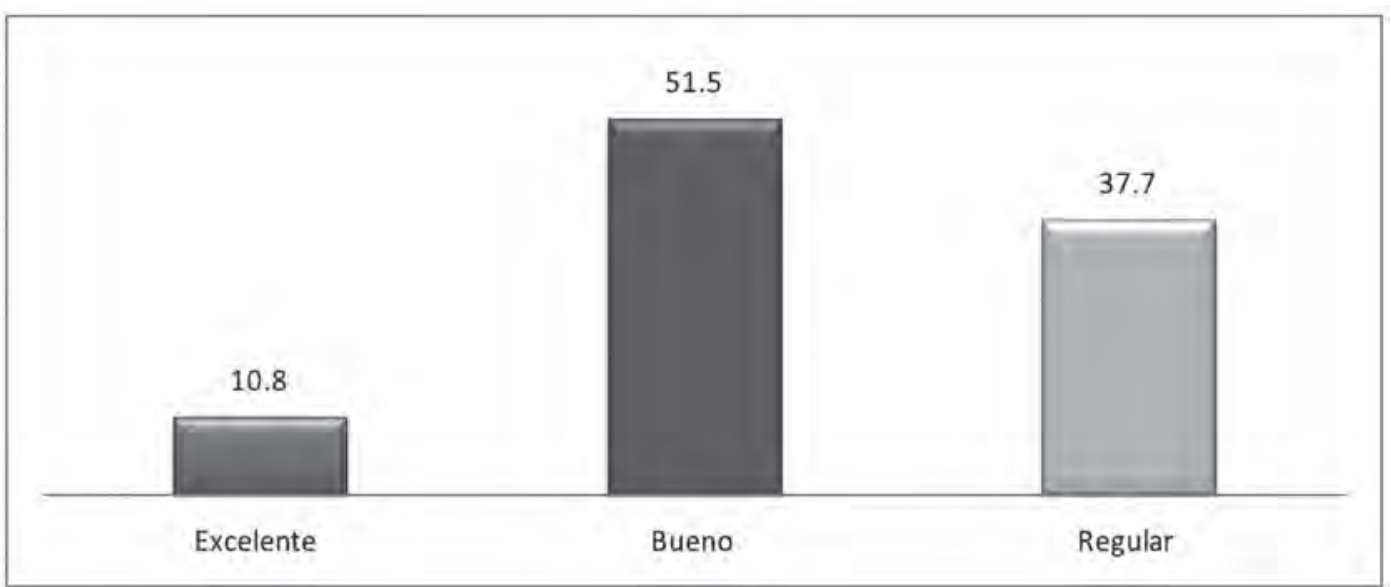

Figura 2

Nivel de rendimiento académico en las prácticas clínicas de los estudiantes de enfermería de una universidad privada de Lima, 2015.

En la figura $\mathrm{N}^{\circ} 2$, según el nivel de rendimiento académico de los estudiantes de enfermería en las prácticas clínicas, el 51,5 \% presenta un rendimiento académico bueno, mientras que el $37,7 \%$ un rendimiento regular y el $10,8 \%$ un rendimiento excelente.

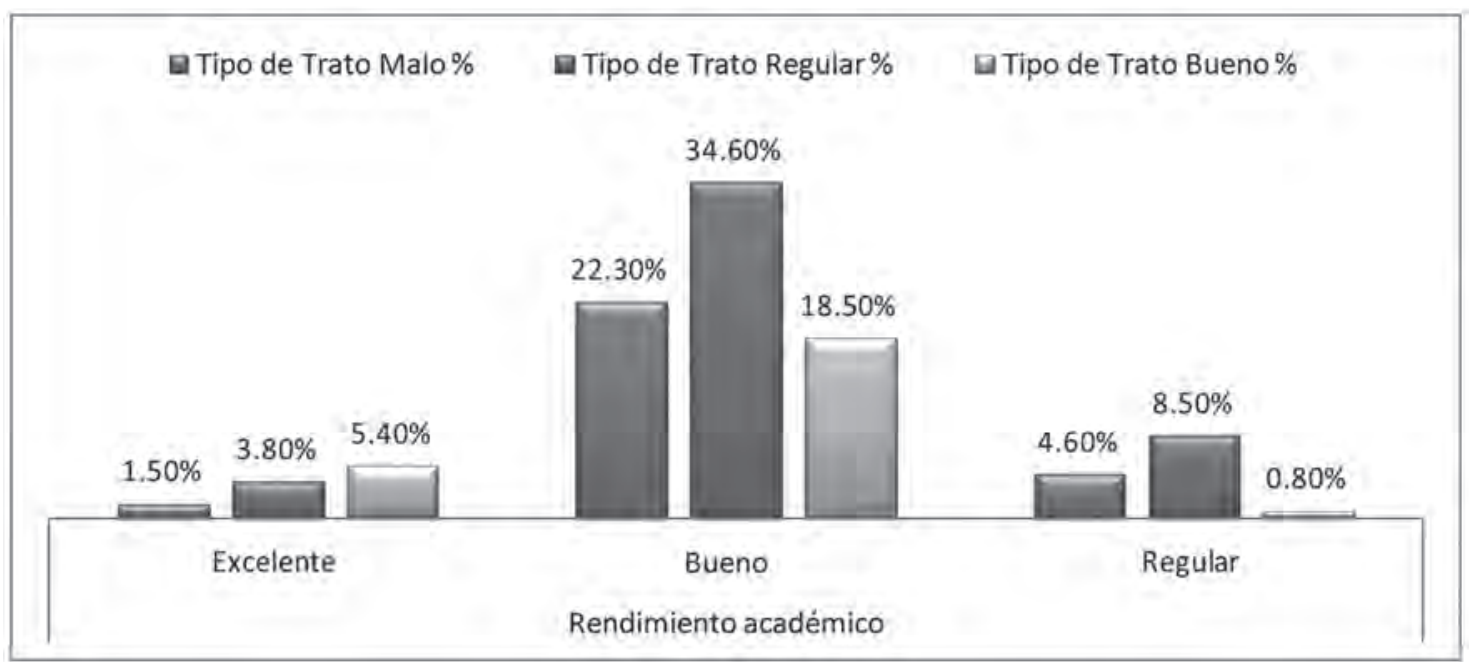

Figura 3

Relación entre el tipo de trato del docente y el rendimiento académico en las prácticas clínicas de los estudiantes de enfermería de una universidad privada de Lima, 2015.

En la figura $\mathrm{N}^{\circ} 3$ se observa que del $100 \%$ de los estudiantes, el $34,6 \%$ presentan un rendimiento académico bueno frente a un trato regular del docente de prácticas clínicas, así mismo el 22,3\% que tienen un buen rendimiento refieren que el docente los trata mal, sin embargo el $18.5 \%$ que reciben un buen trato, presentan un rendimiento de la misma manera. 
Trato del docente y el rendimiento académico en las prácticas clínicas de los estudiantes de enfermería de una Universidad Privada de Lima, 2015

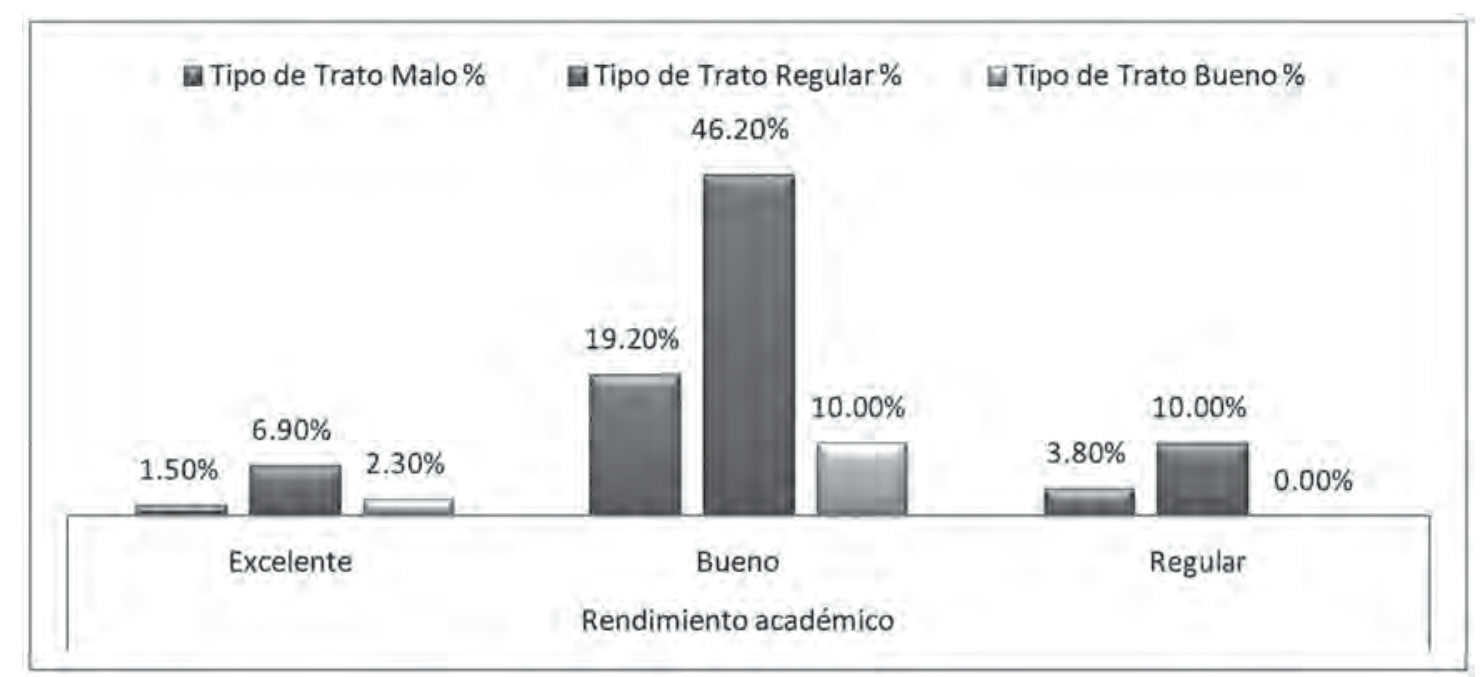

Figura 4

Relación entre el tipo de trato del docente en la dimensión comunicación y el rendimiento académico en las prácticas clínicas de los estudiantes de enfermería de una universidad privada de Lima, 2015.

En la figura 4 se observa que del $100 \%$ de los estudiantes, el 60\% tienen un rendimiento académico bueno frente a una comunicación regular por parte del docente de prácticas clínicas. Por otro lado el 19,2\% que presentan un buen rendimiento dicen que el docente tiene una mala comunicación, no obstante el $10 \%$ de estudiantes que tienen una buena comunicación con el docente, presentan un rendimiento académico bueno.

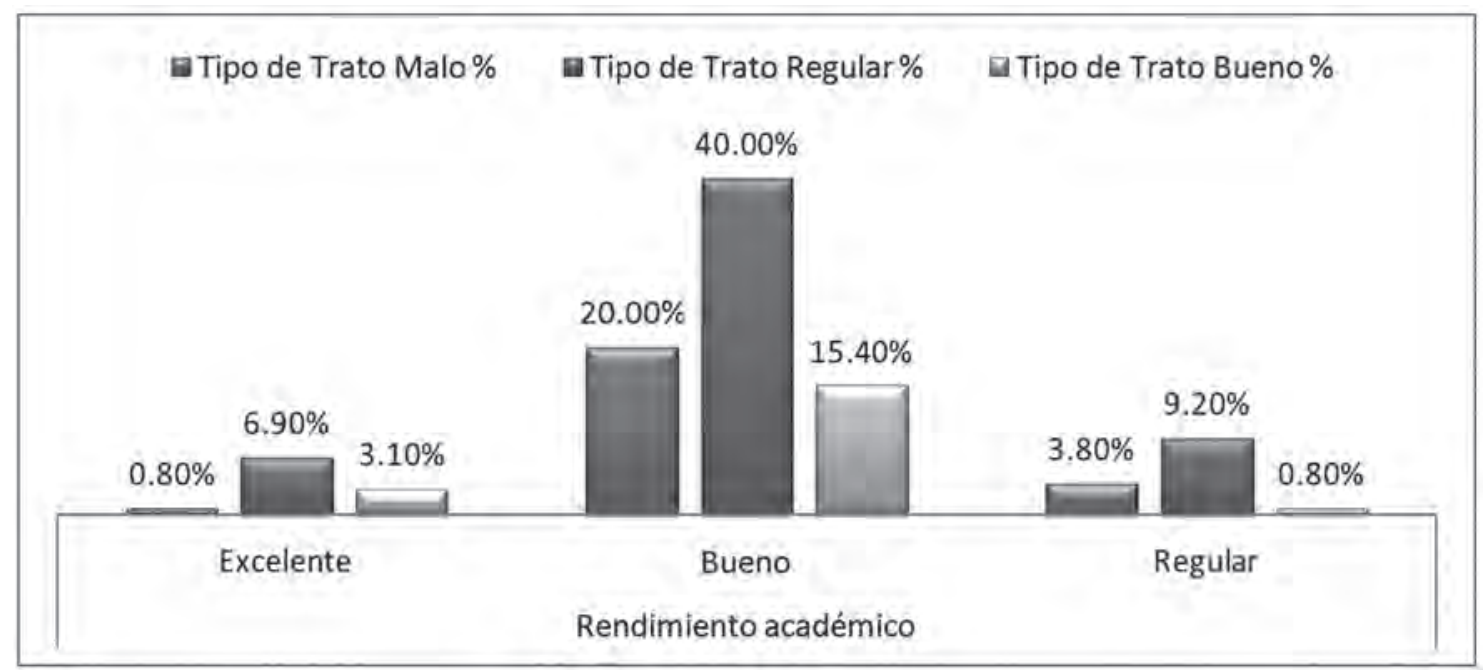

Figura 5

Relación entre el tipo de trato del docente en la dimensión empatía y el rendimiento académico en las prácticas clínicas de los estudiantes de enfermería de una universidad privada de Lima, 2015.

En la figura $\mathrm{N}^{\mathrm{o}} 5$ se observa que del $100 \%$ de los estudiantes, el 40\% refieren una empatía regular por parte del docente de prácticas clínicas y presentan un rendimiento bueno, asimismo el $20 \%$ que tiene un buen rendimiento señalan que el docente no es empático. Por otro lado el $15.4 \%$ de estudiantes que dicen que el docente es empático, presentan un buen rendimiento académico. 


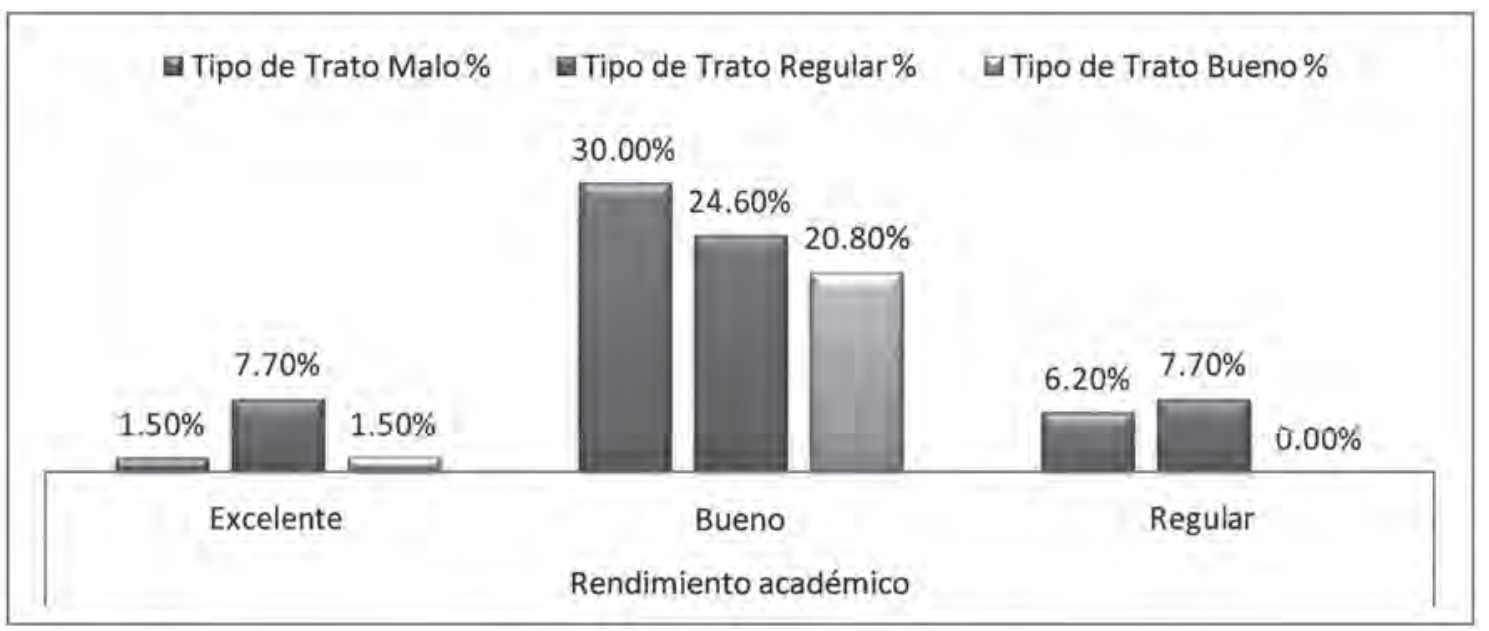

Figura 6

Relación entre el tipo de trato del docente en la dimensión respeto y el rendimiento académico en las prácticas clínicas de los estudiantes de enfermería de una universidad privada de Lima, 2015.

En la figura $\mathrm{N}^{\circ} 6$ se observa que del $100 \%$ de los estudiantes, el $30 \%$ tiene un rendimiento académico bueno frente a la falta de respeto del docente de prácticas clínicas. Asimismo 24,6\% que tienen buen rendimiento, señalan que el docente presenta un respeto regular hacia ellos. No obstante, el 20.8\% que dicen que el docente los respeta, tienen un rendimiento bueno.

Tabla 1

Análisis de correlación entre el tipo de trato del docente según sus dimensiones y el rendimiento académico en las prácticas clínicas de los estudiantes de enfermería de una universidad privada de Lima, 2015

\begin{tabular}{llll}
\hline Tau-b de Kendall & $\mathrm{N}$ & valor & $\mathrm{P}$ \\
\hline En general & 130 & -0.189 & 0.012 \\
Dimensión Comunicación & 130 & -0.129 & 0.082 \\
Dimensión Empatía & 130 & -0.145 & 0.036 \\
Dimensión Respeto & 130 & -0.137 & 0.019 \\
\hline
\end{tabular}

En la tabla 1, se observa que existe una relación inversa (baja) entre el tipo de el trato del docente en forma general y el rendimiento académico $(\mathrm{t}=-.189$; $\mathrm{p}<, 05)$, al igual que en la dimensión empatía $(\mathrm{t}=-.145$; $\mathrm{p}<, 05)$ y respecto $(\mathrm{t}=-.137 ; \mathrm{p}<, 05)$. Por otro lado el trato del docente en la dimensión comunicación y el rendimiento académico no existe relación alguna ( $\mathrm{t}=$ $-.129 ; \mathrm{p}>, 05)$.

\section{DISCUSIÓN}

La enfermería abarca cuatro funciones principales, siendo la docencia una de ellas. Sin embargo en este contexto nos enfocamos al enfermo de prácticas clínicas que asume el rol de docente con los estudiantes que vienen al área asistencial en el cual trabaja el enfermero. Sebold y Carraro (2011) refieren que muchas veces los enfermeros que asumen este rol no tienen conocimiento de lo que es la práctica pedagógica que se necesita para brindar una educación de calidad. En este contexto, el enfermero docente sólo enseña sus propias experiencias prácticas del cuidado, sin tener en cuenta las metodologías innovadoras que propician un mejor aprendizaje. No obstante no involucra que el enfermero no pueda desarrollarse como docente en el área clínica.

Según Aceituno, Estévez y Martínez (2007) señalan que los profesionales de enfermería que realizan la función docente-enfermero son el anexo ente la universidad y el lugar donde realizan sus prácticas clínicas. La enfermera que asume esta responsabilidad 
Trato del docente y el rendimiento académico en las prácticas clínicas de los estudiantes de enfermería de una Universidad Privada de Lima, 2015

es la figura fundamental quien se encargara de integrar al estudiante a su ámbito de trabajo, además enseñara las actividades que desempeña durante su jornada laboral y resolverá sus dudas e inquietudes. Además añade que el rol docente que se ejerce el enfermero en las prácticas clínicas merece ser reconocido e implica la necesidad de adquirir una serie de competencias que no forman parte de la formación de pregrado.

Es imprescindible entonces, que el docente de enfermería adicione el componente axiológico en su actuar, debido a que la calidad en la formación profesional no solo depende de los conocimientos y habilidades sino también de los valores que regulan la actuación profesional ante la sociedad (Sánchez y Gainza, 2010; Díaz, Castro y Cuevas, 2012).

En un estudio realizado en Chile, por los estudiantes mencionaron cuáles eran las cualidades que sobresalen de un docente para ser considerado un buen profesor; obteniendo como resultado la responsabilidad en un 100\%, el respeto en un 89,4\% y la empatía en un 71\% (Cabalín, Navarro, Zamora y San Martín, 2010).

Hay que tener en cuenta, que el éxito educativo de la enseñanza está en su producto y en la Universidad éste puede medirse, en unos casos, en la cantidad de graduados y titulados; pero cuando el producto está en proceso se mide en términos de rendimiento académico de los alumnos (Bellido, 2011). Para Tonconi (2010), el rendimiento académico constituye un indicador del nivel de aprendizaje alcanzado, representa el nivel de eficacia en la consecución de los objetivos curriculares de las diversas asignaturas, es por eso que en el sistema educativo nacional e internacional dan mayor importancia a este indicador.

Los resultados presentados en el estudio evidencian que existe una relación inversa (baja) entre el tipo de "trato del docente" y el "rendimiento académico" $(\mathrm{t}=-.189 ; \mathrm{p}<, 05)$. La relación inversa que se refleja en las variables, se debe a que un $22,3 \%$ de estudiantes que presentan un rendimiento académico bueno señalan que el docente los trata mal y un 1,5\% que dice lo mismo, tiene un rendimiento excelente. Es decir frente un trato inadecuado por parte del docente, el estudiante tiene un rendimiento bueno y excelente. Estos resultados pueden deberse a la resiliencia que presentan estos estudiantes, que es la capacidad de enfrentar situaciones problemáticas, desarrollada mediante la participación activa en la vida familiar (García y Domínguez, 2013). De lo contrario, estaríamos hablando de personas que están acostumbradas a trabajar bajo presión, actuando con eficacia en situaciones de desacuerdo, oposición y diversidad. Entonces podría afirmarse que estas personas tienen la capacidad para responder con alto desempeño en situaciones de mucha exigencia (Alles, 2003). Sin embargo esto no justifica que deba haber un trato inadecuado del docente de prácticas clínicas para obtener un rendimiento bueno o excelente en el estudiante y muchos menos considerar esta manera de ser del docente, como una metodología para que el estudiante se desempeñe mejor.

Por su parte, Bellido (2011) encontró relación significativa en un estudio realizado en la Universidad Nacional del Callao, entre el desempeño docente y el rendimiento académico de los alumnos de la Facultad de Ingeniería. Así mismo, en otro estudio realizado por Monrroy (2012) en un Colegio de Ventanilla, encontró que existe relación entre el desempeño docente y el rendimiento académico en los estudiantes de nivel secundario.

A pesar de las diferentes características de la población de estos tres resultados mencionados, se puede distinguir que el rendimiento académico está asociado significativamente con el docente. Esta realidad, también se evidencia en un estudio realizado en Madrid donde se encontró que las actitudes de los docentes con el estudiante tenían un claro efecto sobre el clima de aprendizaje, además indicaron que la repercusión principal en el estudiante es la motivación o desmotivación (Álvarez, Tabera, Hernando y Rubio, 2013).

Diversos estudios de investigación han coincidido que el trato que brinda el docente al estudiante es un factor asociado al rendimiento académico, de esta manera, convirtiéndose en el agente principal que fomenta y garantiza el aprendizaje durante la enseñanza clínica de los estudiantes de enfermería, facilitando los elementos necesarios para el desarrollo de habilidades y la vinculación entre la teoría y la práctica (Crespo, Gonzales y Agama, 2011). No obstante no se evidencian estudios que abarquen al docente de prácticas clínicas y el rendimiento académico.

Con respecto al tipo de trato del docente de enfermería en las prácticas clínicas, se ha encontrado que a nivel general el trato es regular con el $46,9 \%$, mientras que el $28,5 \%$ de estudiantes dice que es malo y el $24,6 \%$ es bueno. Así mismo el 63,1\% de estudiantes refieren una comunicación regular por parte del docente de prácticas clínicas, el 24,6\% malo y el 12,3\% bueno. De la misma manera, el 56,2\% refieren una empatía regular del docente de prácticas clínicas, el 24,6\% malo y el $19,2 \%$ bueno. Por último el $40 \%$ dicen que el docente tiene un respeto regular, el 37,7\% malo y el $22,3 \%$ bueno. Entonces se puede observar que existe 
una inclinación tanto de manera general como en sus dimensiones del trato regular y malo con respeto al trato del docente de prácticas clínicas.

Estos resultados no son tan esperanzadores como los encontrados por Muñasqui (2007) en otra universidad de Lima, donde el 56\% de estudiantes consideró que el trato del docente en las prácticas clínicas es regular, seguido de un $36 \%$ que manifestó que es bueno y un $8 \%$ que aseguro tener un trato deficiente, es decir en este estudio, casi el $100 \%$ dice que el trato es bueno a regular.

Existen muchos factores que pueden influir en las actitudes del enfermero responsable de prácticas, una de ellas es el estrés laboral y el mal afrontamiento al mismo, la profesión de enfermería requiere de mucha responsabilidad sobre la vida, algunas veces el enfermero que cumple la función docente debido a una mala organización de su tiempo tiene que lidiar con los estudiantes y a la vez los pacientes, convirtiéndose en una carga, pasando a ser la docencia en algo no favorable para el cumplimiento de sus actividades, sin embargo esto no es una justificación para su actuar (Ribera et ál., 1993).

Por otro lado, muchas de estos profesionales, tienen a tener actitudes de autoritarismo por haber sido formadas en la educación tradicional conocida como "vieja escuela", una pedagogía basada en el formalismo, donde la disciplina, las normas y el castigo eran parte fundamental para el aprendizaje, pero que descuida al estudiante como un ser integral e independiente de su aprendizaje (Narváez, 2006).

Pulido (2009) refiere que una de las maneras de medir el trato del docente, es a través de las habilidades sociales que esta evidencia, donde se considera la dimensión de la comunicación: área verbal y no verbal, el respeto y la empatía.

La comunicación en la interacción estudiante-docente es de suma importancia para el fortalecimiento del proceso de enseñanza-aprendizaje. Además el diálogo como forma de comunicación, aporta a la transmisión, transferencia y construcción del conocimiento (Granja, 2013).

Sin embargo en este estudio se observa que no existe relación entre el tipo de trato del docente en la dimensión comunicación y el rendimiento académico $(\mathrm{t}=-.129 ; \mathrm{p}>, 05)$. Estos resultados pueden deberse a que el $60 \%$ de estudiantes presentó un rendimiento académico bueno frente a una comunicación regular por parte del docente de prácticas clínicas. Además el $10 \%$ de estudiantes que tienen una buena comunicación con el docente, presentan un rendimiento académico bueno. (Ver figura 4). Es decir la mayor parte de los estudiantes refieren que existe una buena comunicación con el docente y también presentan un rendimiento académico entre regular y bueno. De los cuales un 19,2\% de los estudiantes que presentan un buen rendimiento y dicen que el docente tiene una mala comunicación.

Los códigos verbales y no verbales reflejados en el párrafo anterior, pueden ser utilizados de manera eficaz o ineficaz en la comunicación en cualquier relación interpersonal (Paes da Silva y Kalil de Freitas, 2003)

Por otro lado, no se encuentran estudios que hayan relacionado estas dos variables, no obstante en un estudio acerca de "Las habilidades sociales que practican los enfermeros docentes de enfermería en la UNMSM” se encontró que la comunicación ocupa un tercer lugar con un $47.8 \%$, el primer y segundo lugar fueron la asertividad (52.2\%) y la toma de decisiones (56.5\%), respectivamente (Zavala, 2002).

Es importante que los enfermeros que asumen el rol docente en las prácticas clínicas reflexionen acerca de la importancia de brindar una buena comunicación, porque sí el docente descuida este aspecto sólo podría aspirar a tener con sus estudiantes una relación trivial que no repercutiría en el aprendizaje (Escobar, 2015).

Con respeto a la relación entre el tipo de el trato del docente en la dimensión empatía y el rendimiento académico se observa que existe una relación inversa baja $(\mathrm{t}=-.145 ; \mathrm{p}<, 05)$. De los cuales el $40 \%$ de estudiantes refieren una empatía regular por parte del docente de prácticas clínicas, sin embargo presentan un rendimiento bueno, así mismo el 20\% que tiene un buen rendimiento señalan que el docente no es empático. Estos resultados evidencian la relación inversa, ya que la sexta parte de estudiantes que refieren que el docente de prácticas tiene una regular y mala empatía presenta un rendimiento académico bueno. Por otro lado el $\mathbf{1 5 . 4 \%}$ de estudiantes que dicen que el docente es empático, presentan un buen rendimiento académico.

Es importante señalar que la empatía del docente es un elemento básico para el aprendizaje (Lara, Aguiar, Cerpa y Núñez, 2009; Gonzalez, Diez, López y Roman, 2010).Para Ortiz (2007) esta es una conducta que nunca debería perder un docente, porque es también considerada la cualidad que ayuda al estudiante a la realización y maduración de sus habilidades, mejorando su desenvolvimiento, esto se debe a que el alumno siente cuando es aceptado por el docente. 
Trato del docente y el rendimiento académico en las prácticas clínicas de los estudiantes de enfermería de una Universidad Privada de Lima, 2015

El docente como líder en su clase propicia a que el alumno pueda adquirir sentimientos de superación, de valor personal, de estimación o todo lo contrario, sentimientos de minusvalía, frustración, apatía e inadecuación (Gónzales, 2015). Entonces al hablar de la empatía en docencia hacemos referencia a ambos actores "docentes y alumnos" dado que la personalidad del profesor y su nivel de relación con los alumnos, depende en gran medida del clima percibido (Álvarez et ál., 2013).

En relación al respeto, se observa que existe una relación inversa (baja) entre el tipo de el trato del docente en la dimensión respeto y el rendimiento académico $(\mathrm{t}=-.137 ; \mathrm{p}<, 05)$. Nuevamente se observa la relación inversa, reflejado en el $30 \%$ de estudiantes que tiene un rendimiento académico bueno frente a la falta de respeto del docente de prácticas clínicas, así mismo 24,6 \% que tienen buen rendimiento, señalan que el docente presenta un respeto regular hacia ellos. No obstante, el $20.8 \%$ que dicen que el docente los respeta, tienen un rendimiento bueno. Por otro lado, no encontramos estudios que relacionen el respeto del docente de prácticas con el rendimiento académico del estudiante. Sin embargo, en un estudio realizado en México por Ríos, Romero y Olivo (2013) los estudiantes manifestaron que tanto en las prácticas clínicas como en el aula de enfermería se evidencia la existencia de rechazo en un 35.4\%, la intimidación en un $38.4 \%$, la humillación pública en un $16.3 \%$, la violencia verbal en un $28 \%$, el acoso sexual en un $8.3 \%$ y la agresión física en un $0.8 \%$, estos resultados reflejan como el respeto hacia los estudiantes se ha ido degradando en el campo clínico.

Al buscar las causas por las que el docente ha perdido el respeto a los alumnos, no vamos encontrar mucha información al respecto, sin embargo, tomar en cuenta a lo que consideran los alumnos como falta de respeto, nos ayuda a aclarar este panorama.

No se debe olvidar que el respeto es una de las bases sobre la cual se sustenta la ética y la moral en cualquier campo y en cualquier época. Klein (2011) refiere que el respeto es una característica que evidencia la buena relación que existe entre el docente y el estudiante. Consideramos entonces, que el conocimiento se genera a partir del buen ejemplo, estas tres dimensiones mencionadas, favorecen la reflexión del saber y la relación en el ámbito clínico, llegando a cuestionarse el significado educativo de la función del docente en el campo práctico y el desempeño académico.

El desempeño académico se denomina también como rendimiento académico, la cual no deja de ser complejo desde su conceptualización (Navarro, 2003). El rendimiento académico de la población en estudio se ve evidenciado en la figura 2, mostrando que el 51,5\% presenta un rendimiento académico bueno en las prácticas clínicas, el 37,7\% es regular y el 10,8\% es excelente. Donde el $100 \%$ de estudiantes tienen un rendimiento académico entre regular, bueno y excelente, no evidenciándose un rendimiento malo.

Estos resultados son distintos a los encontrados por Serón (2006) en un estudio realizado en la Universidad Mayor de San Marcos donde, del 100\% de estudiantes de enfermería, el 59,3\% presentaron un rendimiento académico regular, un $26 \%$ bueno y un $15 \%$ malo. Según la teoría multifactorial del rendimiento académico, se explica que éste, es concebido como un resultado del proceso de enseñanza- aprendizaje por la existencia de varios factores, pero se atribuye que está íntimamente relacionado a la calidad de enseñanza (Bellido, 2011). Sin embargo, el docente no es el único factor determinante de la formación de los estudiantes, ni en su rendimiento académico, justificando así la relación encontrada en nuestro estudio (Rueda, Alonso, Guerra y Martínez, 2014).

A pesar de no ser el único factor en el rendimiento del estudiante, el docente que se involucra con el cuidado, sabe que será capaz de ayudar al otro a crecer a su propio ritmo, y será responsable del crecimiento y desarrollo de sus estudiantes (Waldow, 2009). Así, la relación de la enfermera docente con el estudiante no es de imposición, sino de convivencia; de intervención, de interacción, para poder asumir su propia existencia y trascender en su ser (Terra, Padoin, Gonçalves, Santos y Erdmann, 2008).

La enfermera docente debe de cuidar las necesidades del estudiante de una manera funcional. Este cuidado para con el estudiante no requiere muchas cualidades, se basa en servicio, del cual radica "el ser" de la enfermería, así como el servicio que brinda al paciente con la finalidad de hacer el bien. Ello la lleva a considerar a los estudiantes como "seres" de las cuales debe de cuidar. En este caso el estudiante no es objeto de servicio, sino de interés (Martini, 2010).

\section{Declaración de financiamiento y de conflicto de intereses:}

El estudio fue financiado por los autores, quienes declaran no tener algún tipo de conflicto de interés en la investigación realizada. 


\section{Correspondencia:}

Katherine Mescua Fasanando. Coordinadora de Especialidades de Enfermería, Unidad de Posgrado de Ciencias de la Salud, Universidad Peruana Unión, Carretera Central Km. 19.5 Ñaña, Lima, Perú. e-mail: katherine.mescua@upeu.edu.pe

\section{REFERENCIAS BIBLIOGRÁFICAS}

Aceituno, M., Estévez, M., \& Martínez, M. (2007). "Competencias de enfermería del rol investigador y tutorización de alumnos: grado de desarrollo en unidades de hospitalización”. Retrieved from http://docplayer. es/7326193 Escuela universitaria de enfermeria santa madrona de la fundacion la caixa.html

Alles, M (2003). Gestión por competencias: El diccionario. Buenos Aires: Editorial Granica Álvarez, M., Tabera, M., Hernando, A., \& Rubio, M. (2013). Percepción de los estudiantes Universitarios sobre las actitudes de los docentes y su influencia en el clima de aprendizaje. Retrieved from http://abacus.universidadeuropea.es/ bitstream/handle/11268/1966/1616.pdf?sequence=1

Bellido, R. (2011). Relación entre desempeño docente y rendimiento académico en la Escuela Profesional de Ingeniería Pesquera y de Alimentos de la Universidad Nacional del Callao. Retrieved from http://www.unac. edu.pe/documentos/organizacion/vri/cdcitra/Informes Finales Investigacion/Marzo 2011/IF BELLIDO FLORES FIPA.PDF

Bettancourt, L., Muñoz, L. A., Aparecida, M., \& Merighi, B. (2011). El docente de enfermería en los campos de práctica clínica: un enfoque fenomenológico, 19(5), 9. Retrieved from http://www.scielo.br/scielo.php?script=sci arttext\&pid=S0104- $\quad$ 11692011000500018\&lng=en . http://dx.doi.org/10.1590/S0104-11692011000500018.

Cabalín, D., Navarro, N., Zamora, J., \& San Martín, S. (2010). Concepción de Estudiantes y Docentes del Buen Profesor Universitario: Facultad de Medicina de la Universidad de La Frontera. International Journal of Morphology, 28(1), 283-290. http://doi.org/10.4067/ S0717-95022010000100042

Cortez, E. (2008). Docencia de Enfermería. Recuperado de: http://docenciaenenfermeria.blogspot.pe/2008/08/ docencia-en-enfermeria.html

Crespo, S., Gonzales, M., \& Agama, A. (2011). Percepción de los alumnos de Enfermería sobre los factores que inciden en su aprendizaje clínico, 14(2), 88-93.

Díaz, M., Castro, D., \& Cuevas, B. (2012). Valores profesionales de enfermería: Una mirada hacia la formación en la Educación Superior. Humanidades Médicas, 12(2), 289-299. Retrieved from http://scielo.sld.cu/scielo. php?pid=S1727-81202012000200011\&script=sci_arttext

Escobar, M. (2015). Influencia de la interacción alumnodocente en el proceso enseñanza- aprendizaje. Revista de tecnología y sociedad. (8)(5). ISSN: 2007-3607. Recuperado de: http://www.udgvirtual.udg.mx/paakat/ index.php/paakat/article/view/230/347

García, M., \& Domínguez, E. (2013). Desarrollo teórico de la Resiliencia y su aplicación en situaciones adversas : Una revisión analítica. Revista Latinoamericana de
Ciencias Sociales, Niñez y Juventud, 11(1), 63-77. http:// doi.org/10.11600/1692715x.1113300812

Gónzales, F. (2015). El buen humor y la risa en la práctica docente en el aula universitaria.

Retrieved from http://unimex.edu.mx/Investigacion/ DocInvestigacion/El buen humor y la risa en la practica docente en el aula universitaria.pdf

Gonzalez, M., Diez, M., López, F., \& Roman, M. (2010). La importancia del clima emocional del aula desde la perspectiva del alumnado universitario. Revistas de Enseñanza Universitaria, 35, 16-27. Retrieved from http://institucional.us.es/revistas/universitaria/35/art_2. pdf

Granja, C. (2013). Caracterización de la comunicación pedagógica en la interacción. Revista de Investigación En enfermería: Imagen y Desarrollo, 15, 65-93.

Klein, F. (2011). Las tensiones en la relación docentealumno: Una investigación del ámbito educativo. Revista de Ciencias Sociales, 1-28. Retrieved from http:// apostadigital.com/revistav3/hemeroteca/fklein3.pdf

Lara, A., Aguiar, M., Cerpa, G., \& Núñez, H. (2009). Relaciones docente-alumno y rendimiento académico: Un caso del Centro Universitario de Ciencias Exactas e Ingenierías de la Universidad de Guadalajara. Sinéctica. Retrieved from http://www.scielo.org.mx/scielo. php?pid=S1665-109X2009000200006\&script $=$ sci arttext

Lescano, E. (2002). Interacción entre el desempeño docente y las condiciones de estudio sobre el rendimiento académico de los estudiantes de la Universidad Nacional de Piura.

Martini, J. (2010). El currículo y la formación del enfermero. Revista Brasileira de Enfermagem, 61, 409.

Monrroy, M. (2012). Desempeño Docente y Rendimiento Alumnos de una Institución Educativa de Ventanilla Callao. Retrieved from http://repositorio.usil.edu.pe/wpcontent/uploads/2014/07/2012 Monrroy Desempeño docente y rendimiento académico en matemática de los alumnos de una institución educativa de Ventanilla Callao.pdf

Montero, E. y Villalobos, J. (2004). Estudio comparativo del promedio de admisión a la Universidad de Costa Rica y sus componentes: Aplicación del año 2001. San José, Costa Rica: SIEDIN.

Muñasqui, I. (2007). Percepción de los estudiantes de enfermería, UNMSM sobre el trato del docente al estudiante durante la práctica clínica en la asignatura enfermería en la salud del adulto y anciano.

Narváez, E. (2006). Una mirada a la escuela nueva. Educere, 10(35), 629-636. Recuperado en 15 de febrero de 2016, 
Trato del docente y el rendimiento académico en las prácticas clínicas de los estudiantes de enfermería de una Universidad Privada de Lima, 2015

de http://www.scielo.org.ve/scielo.php?script=sci_arttext\&pid=S1316- $\quad 49102006000400008 \& \operatorname{lng}=$ es\&tln$\mathrm{g}=\mathrm{es}$.

Navarro, R. E. (2003). REICE - Revista Electrónica Iberoamericana sobre Calidad, Eficacia y Cambio en Educación 2003, Vol. 1, No. 2 http://www.ice.deusto.es/rinace/ reice/vol1n2/Edel.pdf, 1(2), 1-15.

Ortiz, G. (2007). Realización profesional Docente. México: Editorial Progreso. Recuperado de:https:// books.google.com.pe/books?id=NBKd7ys1cFwC\&p$\mathrm{g}=\mathrm{PA} 45 \& \mathrm{lpg}=\mathrm{PA} 45 \& \mathrm{dq}=$ empatia en el trato con el docente\&source $=$ bl\&ots $=$ HHmzTMz3OD\&sig $=$ ryMVQQT4fUOUZjUKnrMs0APSVw0\&hl=es\&sa=X\&ved=0ahUKEwj396uaoLvKAhXLjpAKHaG1A9gQ6AEIIDAB\# $\mathrm{v}=$ onepage $\& \mathrm{q} \& \mathrm{f}=$ false

Paes da Silva, J., \& Kalil de Freitas, R. (2003). Influencias del comportamiento comunicativo verbal y no verbal del Docente en el aula:Visión de los alumnos y docentes de Enfemería. Revista de Enfemería Global, 1-11.

Pulido, I. (2009). “Habilidades sociales del docente," 1-9. Retrieved from http://www.csi- csif.es/andalucia/modules/mod ense/revista/pdf/Numero 25/INMACULADA PULIDO 2.pdf

Ribera, D., Cartagena, E., Reig, A., Romá, M., Sans, I., \& Caruana, A. (1993). Estrés laboral y salud en profesionales de enfermería.

Ríos, B., Romero, S., \& Olivo, R. (2013). La percepción del maltrato de las y los estudiantes nayaritas en las carreras universitarias de medicina y enfermería. Un primer acercamiento, (9), 20-30.

Rueda, M., Alonso, A., Guerra, M., \& Martínez, M. (2014). El contexto: factor clave en el desarrollo de la docencia en la universidad. Revista Argentina de Educación Superior, 9, 9-36. Retrieved from http://www.revistaraes.net/ revistas/raes9 art1.pdf

Sánchez, M., \& Gainza, C. (2010). Valores: Esencia de la Enfermera. Revista Educación en Valores, 1, 80-93. Retrieved from http://servicio.bc.uc.edu.ve/multidisciplinarias/educacion-en-valores/v1n13/art11.pdf
Scherer, Z., \& Scherer, E. (2007). Reflexiones sobre la Enseñanza de Enfemería en la Postmodernidad y la metáfora de una laguna Teórico- Práctica. Revista Latino Enfemagem, 15(3), 1-4.

Sebold, L. F., \& Carraro, T. E. (2011). La práctica pedagógica del docente en enfermería: una revisión integradora de la literatura. Enfermería Global, 10(22), 1-12. http://doi. org/10.4321/S1695-61412011000200023

Serón, N. (2006). Relación que existe entre factores estresantes y rendimiento académico de los estudiantes de Enfermería de la U.N.M.S.M. Retrieved from http:// cybertesis.unmsm.edu.pe/bitstream/cybertesis/1009/1/ Seron_cn.pdf

Terra, M., Padoin, S., Gonçalves, L., Santos, E., \& Erdmann, L. (2008). O dito e o não-dito do ser-docente-enfermeiro/a na compreensão da sensibilidade. Revista Brasileira de Enfermagem, 61(5), 558-564. http://doi.org/10.1590/ S0034-71672008000500005

Tonconi, J. (2010).Factores que influyen en el rendimiento académico y la deserción de los estudiantes de la facultad de Ingeniería Económica de la UNA-PUNO, periodo 2009.

Waldow, R. (2009). Enseñanza de enfermería centrada en el cuidado. Aquichan, 9(3), 246-256.

Yarasca, K. (2012). Perfil de liderazgo transformacional en estudiantes y docentes de práctica de enfermería de la UNMSM según opinión de los estudiantes de la EAP de Enfermería de la UNMSM, Lima - Perú, en el año 2011.

Zavala, M. (2002). Habilidades sociales que practican los enfemeros docentes del Departamento Académico de Enfermería de la UNMSM. Universidad Nacional Mayor de San Marcos.

Recibido: 13/03/2016

Aceptado: 04/05/2016 